\title{
Cyclodextrin Based Biocompatible Nanosystems for Improving Drug Delivery
}

\author{
Lokesh Adhikari $^{1}$ - Nitin Kumar ${ }^{1}$ Abhijit Saha ${ }^{2}$ Mona Semalty $^{1}$ Ajay Semalty ${ }^{1 *}$ \\ ${ }^{1}$ Department of Pharmaceutical Sciences, H.N.B. Garhwal University (A Central University) \\ Srinagar Garhwal- 246174, India \\ ${ }^{2}$ UGC-DAE Consortium for Scientific Research, Kolkata Centre, 3/LB8, plot 8, Bidhan Nagar, Kolkata 700091, \\ India
}

*Corresponding Author: semaltyajay@gmail.com

\author{
Received: 2.8.2021; Revised: 29.8.2021; Accepted: 25.9.2021 \\ CSociety for Himalayan Action Research and Development
}

\begin{abstract}
Cyclodextrins (CDs) have a hollow bucket like conformations with hydrophobic interior and hydrophilic outer surfaces. These structures are well known in macro-molecular chemistry for their unique ability to form inclusion complexes with different organic molecules. These host-guest complexes were presented in papers for decades for improving solubility, permeability, stability and masking toxic effects of drugs. In the present study, we have designed the nanoparticles of $\beta C D$ and HP $\beta C D$, using Pluronic F108 as cosurfactants for improvement in drug delivery using herbal hydrophobic drug curcumin as a model. And formulated nanosystems evaluated for average particle sizing, polydispersity index, zeta potential, ATR-FTIR, per cent encapsulation efficiency, in-vitro permeation and drug solubility. Both the systems produced colloidal solutions of particles size below $384.2 \mathrm{~nm}$ with polydispersity index below 0.682 , the zeta potential of -58.5 and -8.81, per cent encapsulation efficiency up to $13.5 \%$ and an increase in permeation and solubility up to 10 and 7 folds. Observed results of the present studies suggest that biocompatible, non-toxic nanosystems can be developed with different cyclodextrins to improve drug delivery.
\end{abstract}

Keywords: Nanoparticles, Cyclodextrin, Self-Assembly, Curcumin

\section{Introduction}

Cyclodextrins, oligosaccharides are the group of structurally identical natural compounds comprised of (a-1-4) linked $\alpha-D$ glucopyranose units. These are also named cyclomaltoses, cycloamylose and Schrodinger dextrins. Three different parent cyclodextrins are based on several glucopyranose units named; $\alpha, \beta$ and $\gamma$ cyclodextrin. $\alpha$-cyclodextrin is composed of $6, \beta$ of 7 and $\gamma$ of 8 glucopyranose units. The digestion of starch produces these by the enzyme cyclomaltodextrin glucanotransferase. Molecules of cyclodextrins are doughnut- shaped with a hydrophobic inner cavity and hydrophilic outer surface.

Due to the unique shape and structure of cyclodextrin molecules, they tend to trap the molecule of a guest compound inside its cavity as a container; this brings about the formation of inclusion complexes. These types of inclusion complexes studied well for long times in pharmaceutical aids for improving the aqueous solubility, rate and extent of permeability, improving stability and masking the taste and odour of the guest molecule.

As cyclodextrin molecules have good aqueous solubility, they produced the true solution in 
water. Recently it has been discovered that cyclodextrins also have self-assembling properties by which, on the addition of them into the water in an amount more significant than the solubility, cyclodextrin molecules start self-assemble and produce nanoaggregates and then large aggregates.

In the present paper, we have highlighted the production of nanoaggregates of host-guest inclusion complexes. Two systems are produced, one composed of the parent $\beta$ cyclodextrin and the other one consists of derivatized $\mathrm{HP} \beta C D$, using hydrophobic curcumin as a model drug. Formulation evaluated for improvement in the delivery of curcumin.

\section{Material and Methods}

Curcumin is obtained from TCI Chemicals (India) Pvt. Ltd., Sodium Dodecyl Sulphate (SDS) and Hydroxypropyl- $\beta$-cyclodextrin (HP $\beta C D)$ from Central Drug House (P) Ltd. $\beta$ cyclodextrin $(\beta C D)$ from HiMedia Laboratories and Pluronic F108 from Sigma Aldrich, India.

\section{Methodology}

\section{Standard curve preparation}

The standard curve of curcumin was developed by preparing a series of standard solutions of drug concentration of $1,2,3,4,5,6,7,8,9,10 \mathrm{mg} / \mathrm{mL}$ in methanol. $\lambda_{\max }$ was analyzed by scanning a 200 to $1100 \mathrm{~nm}$ solution using EI UV/Visible Spectrophotometer 2375. The curve was plotted between the concentration of Drug in solution and absorbance at $424 \mathrm{~nm}$.

\section{Preparation of nanoparticles}

Direct preparation method with $\beta$ cyclodextrin: Accurately weighed Drug, $\beta$ cyclodextrin and SDS (sodium dodecyl sulphate) in the molar ratios of 1:10:5 dissolved in $100 \mathrm{~mL}$ of distilled water with constant stirring. The isotropic solution was produced by heating it and concentrated to 5\% $w / v$. The solution was then incubated for 24 hours at room temperature for self-assembly of $\beta C D / S D S$ nanoparticles. Produced samples are then stored at $4^{\circ} \mathrm{C}$ for further studies (Li et al., 2016).

Dilution method with HPßCD: In the preparation of nanoparticles, Drug and Pluronic F108 were accurately weighed in the molar ratio of $1: 2$ and dissolved in $50 \mathrm{~mL}$ of distilled water with constant stirring. HP $\beta C D$ equivalent to 4 times in molar ratios was dissolved in $30 \mathrm{~mL}$ of distilled water. Then prepared transparent solution of HP $\beta C D$ was slowly added into a Drug/pluronic solution with constant stirring and heating. Produced solutions were then cooled overnight. Samples were stored in refrigerated conditions for further studies (Perry et al., 2011).

\section{Characterization of Nanoparticles}

Sizing and PDI measurement: At room temperature, particle size and polydispersity index of samples were measured with the angle of detection at $90^{\circ}$ by the use of photon correlation spectroscopy or particle size 
analyzer by Zetasizer Nano ZS90 (Malvern Panalytical Ltd., Malvern, UK.) (Rizvi and Saleh, 2018).

Zeta potential: Zeta cuvettes were filled with samples, and the surface charge was determined at $25^{\circ} \mathrm{C}$ by Zetasizer (Gaikwad et al., 2019).

Table 1. Composition table of curcumin loaded nanoparticles of $\beta-C D$ and HP $\beta-C D$

\begin{tabular}{lccccc}
\hline Formulation & $\begin{array}{c}\text { Curcumin } \\
(\mathbf{m M})\end{array}$ & $\begin{array}{c}\boldsymbol{\beta}-\mathrm{CD} \\
(\mathbf{m M})\end{array}$ & $\begin{array}{c}\text { HPß-CD } \\
(\mathbf{m M})\end{array}$ & $\begin{array}{c}\text { SDS } \\
(\mathbf{m M})\end{array}$ & $\begin{array}{c}\text { Pluronic } \\
\text { F108 }(\mathbf{m M})\end{array}$ \\
\hline F1 & 1 & 10 & - & 5 & - \\
F2 & 1 & - & 10 & - & 2 \\
\hline
\end{tabular}

Percent encapsulation efficiency: All the samples were centrifuged at $10000 \mathrm{rpm}$ at room temperature for 120 mins. to settle the nanoparticles. Supernatant was analyzed for

$$
\text { Encapsulation Efficiency }(\%)=\frac{\text { Initial Concentration }- \text { Final Concentration }}{\text { Final Concentration }} \times 100
$$

\section{Final Concentration}

ATR-FTIR spectroscopy: A small sample was directly placed over diamond ATR element attached to Agilent technologies

Cary 630 FTIR and graph was plotted from 650 to $4000 \mathrm{~cm}^{-1}$ (López-Lorente and Mizaikoff, 2016).

Permeation studies: In-vitro permeation studies were performed by using Franz diffusion cell. A drug solution was taken in the donor compartment and receptor compartment filled with a phosphate buffer solution of $\mathrm{pH}$ 7.4. Cellophane membrane was placed between the receptor and donor compartment. Magnetic bead dropped down in receptor compartment and placed over magnetic stirrer. Pre-heated distilled water at $37^{\circ} \mathrm{C}$ is drug concentration and values placed in the equation given below (Krauland and Alonso, 2007; Shahsavari et al., 2014). continuously injected into the receptor compartment jacket to maintain the temperature. Samples were taken out and replaced with PBS at a definite interval of time for 8 hours. All the samples were then analyzed for drug content with a UV-Visible spectrophotometer (Friend, 1992; Liu et al., 2015).

\section{Results and Discussion}

Sizing and PDI measurement: Particle size of formulation F1 and F2 was determined as 384.2 and $283.7 \mathrm{~nm}$ (Figure 1. (A)). The release of Drug and solubility of nanoparticles majorly depends on the size of particles. 
Smaller particles have higher drug release and more solubility. (Bhatia, 2016).

The polydispersity index of nanoparticles expresses the size uniformity in nanoparticles. Polydispersity index for formulation F1 and F2 was found to be 0.682 and 0.566 (Figure 1. (A)). As F2 showed the smaller polydispersity index, indicating that a large number of particles was in the same size range (Jain et al., 2018).

Zeta Potential: The surface charge over the particles present in formulations F1 and F2

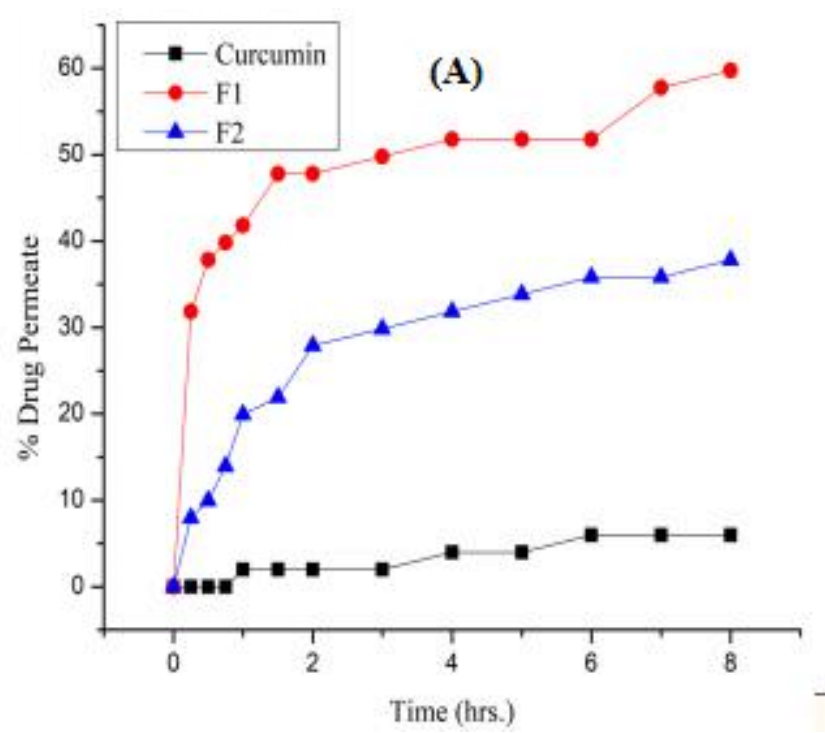

was found to be -8.81 and $-58.5 \mathrm{mV}$ (Figure 1 . (A)). Zeta potential value is directly proportional to the stability of nanoparticles as the rate of agglomeration of particles is dependent on surface charge. Since formulation F2 had a higher zeta potential value, therefore this formulation could be more stable than F1 ("Evaluation Methods for Properties of Nanostructured Body," 2018; Gaikwad et al., 2019).

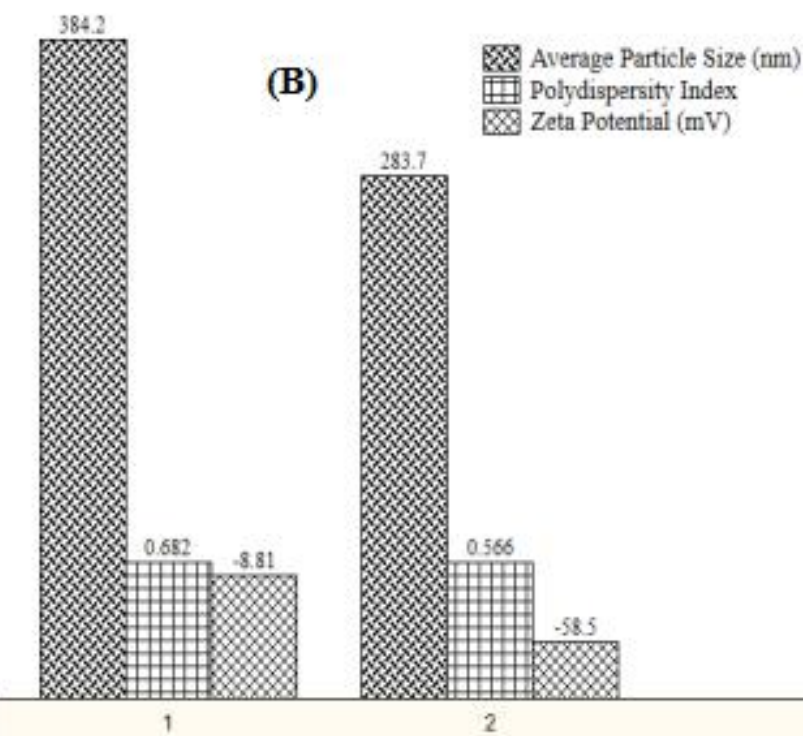

Figure 1 (A): Comparative in-vitro permeation study plot for free drug and formulations, (B): DLS data of formulations

Solubility: Aqueous solubility of curcumin was enhanced by 7 and 6.3 folds in formulation F1 and F2. Aqueous solubility of Drug is increased because of the formation of drug-cyclodextrin inclusion complexes. The presence of smaller particles and more soluble $\mathrm{HP} \beta C D$ in formulation F1 showed higher solubility than formulation F2 (Incecayir, 2015; Saokham et al., 2018).
Encapsulation Efficiency: The percent encapsulation efficiency of formulation $\mathrm{F} 1$ and F2 was found to be 0.934 and $13.574 \%$. Formulation F2 had greater per cent encapsulation efficiency, which may be due to the inclusion complexation of Drug with HP $\beta C D$. (Shahsavari et al., 2014). 


\section{ATR-FTIR Spectroscopy: ATR-FTIR} spectrum of all the samples and excipients suggests the formation of host-guest inclusion complexes ("IR Spectrum Table \& Chart," n.d.; López-Lorente and Mizaikoff, 2016).

Permeation studies: In-vitro permeation of Drug improved by 6 and 10 folds for formulations F1 and F2 compared to free Drug as shown in Figure 1. (B). Drug permeation in formulations is enhanced due to smaller particle size and improved solubility by cyclodextrin complexation (Creasey et al., 1978; Martinez and Amidon, 2002).

Table 2. Solubility comparison of formulations with pure Drug

\begin{tabular}{cc}
\hline Formulations & $\begin{array}{c}\text { Solubility } \\
(\mathbf{m g} / \mathbf{m L})\end{array}$ \\
\hline Curcumin & 0.003 \\
F1 & 0.021 \\
F2 & 0.019 \\
\hline
\end{tabular}

\section{Conclusion}

Both parent and derivatized cyclodextrin complexes have been reported to produce biocompatible nano systems. These nanoaggregates have also been reported to facilitate the effectiveness of low bioavailability herbal components by increasing drug solubility, permeation, and minimizing particle size, among other things. Co-surfactants can also change the size of nanoaggregates, their surface charge, and their solubility profile. Because cyclodextrins are cheap and readily available, non-toxic, and have been investigated for a long time, these biodegradable nano systems give ample future research space.

\section{References}

Bhatia, S., 2016. Nanoparticles Types, Classification, Characterization, Fabrication Methods and Drug Delivery Applications, in: Natural Polymer Drug Delivery Systems. Springer International Publishing, Cham, pp. 33-93. https://doi.org/10.1007/978-3-319-411293_2

Creasey, N.H., Battersby, J., Fletcher, J.A., 1978. Factors affecting the permeability of skin. The relation between in vivo and in vitro observations. Curr. Probl. Dermatol. 7, 95-106.

Evaluation Methods for Properties of Nanostructured Body, 2018. in: Nanoparticle Technology Handbook. Elsevier, pp. 301-363. https://doi.org/10.1016/B978-0-44464110-6.00006-8

Friend, D.R., 1992. In vitro skin permeation techniques. J. Controlled Release 18, 235-248. https://doi.org/10.1016/01683659(92)90169-R

Gaikwad, V.L., Choudhari, P.B., Bhatia, N.M., Bhatia, M.S., 2019. Chapter 2 Characterization of pharmaceutical nanocarriers: in vitro and in vivo studies, in: Grumezescu, A.M. (Ed.), Nanomaterials for Drug Delivery and Therapy. William Andrew Publishing, pp. 33-58. https://doi.org/10.1016/B978-012-816505-8.00016-3

Incecayir, T., 2015. The effects of surfactants on the solubility and dissolution profiles of a poorly water-soluble basic drug, carvedilol. Pharm. 70, 784-790.

IR Spectrum Table \& Chart [WWW Document], n.d.. Sigma-Aldrich. URL https://www.sigmaaldrich.com/technical- 
documents/articles/biology/ir-spectrumtable.html (accessed 9.12.19).

Jain, R., Savla, H., Naik, I., Maniar, J., Punjabi, K., Vaidya, S., Menon, M., 2018. Chapter 33 - Novel Nanotechnology Based Delivery Systems for Chemotherapy and Prophylaxis of Tuberculosis, in: Mustansar Hussain, C. (Ed.), Handbook of Nanomaterials for Industrial Applications, Micro and Nano Technologies. Elsevier, pp. 587-620. https://doi.org/10.1016/B978-0-12813351-4.00034-1

Krauland, A., Alonso, M., 2007. Chitosan/cyclodextrin nanoparticles as macromolecular drug delivery system. Int. J. Pharm. 340, 134-142. https://doi.org/10.1016/j.ijpharm.2007.03. $\underline{005}$

Li, Z., Li, H., Wang, C., Xu, J., Singh, V., Chen, D., Zhang, J., 2016. Sodium dodecyl sulfate/ $\beta$-cyclodextrin vesicles embedded in chitosan gel for insulin delivery with $\mathrm{pH}$-selective release. Acta Pharm. Sin. B, Functional Materials, Nanocarriers, and Formulations for Targeted Therapy 6, 344-351. https://doi.org/10.1016/j.apsb.2016.03.00 3

Liu, D., Zhang, C., Zhang, X., Zhen, Z., Wang, P., Li, J., Yi, D., Jin, Y., Yang, D., 2015. Permeation measurement of gestodene for some biodegradable materials using Franz diffusion cells. Saudi Pharm. J. 23, 413-420. https://doi.org/10.1016/j.jsps.2015.01.012

López-Lorente, Á.I., Mizaikoff, B., 2016. Recent advances on the characterization of nanoparticles using infrared spectroscopy. Trac Trends Anal. Chem., Recent advances and trends in Analytical Nanoscience and Nanotechnology 84, 97106.

https://doi.org/10.1016/j.trac.2016.01.012
Martinez, M.N., Amidon, G.L., 2002. A mechanistic approach to understanding the factors affecting drug absorption: a review of fundamentals. J. Clin. Pharmacol. 42, 620-643. https://doi.org/10.1177/009700020420060 05

Perry, C., Hébraud, P., Gernigon, V., Brochon, C., Lapp, A., Lindner, P., Schlatter, G., 2011. Pluronic and $\beta$-cyclodextrin in water: from swollen micelles to selfassembled crystalline platelets. Soft Matter 7, 3502-3512. https://doi.org/10.1039/C0SM01092H

Rizvi, S.A.A., Saleh, A.M., 2018. Applications of nanoparticle systems in drug delivery technology. Saudi Pharm. J. 26, 64-70. https://doi.org/10.1016/j.jsps.2017.10.012

Markham, P., Muankaew, C., Jansook, P., Loftsson, T., 2018. Solubility of Cyclodextrins and Drug/Cyclodextrin Complexes. Mol. Basel Switz. 23. https://doi.org/10.3390/molecules230511 61

Shahsavari, S., Vasheghani-Farahani, E., Ardjmand, M., Dorkoosh, F., 2014. Design and Characterization of Acyclovir Loaded Nanoparticles for Controlled Delivery System. Curr. Nanosci. 10, 521531.

https://doi.org/10.2174/157341371130966 60128

Viswanathan, P., Muralidaran, Y., Ragavan, G., 2017. Chapter 7 - Challenges in oral drug delivery: a nano-based strategy to overcome, in: Andronescu, E., Grumezescu, A.M. (Eds.), Nanostructures for Oral Medicine, Micro and Nano Technologies. Elsevier, pp. 173-201. https://doi.org/10.1016/B978-0-32347720-8.00008-0 